\title{
Una etnografía audiovisual del entrenamiento en natación sincronizada: un proceso de trabajo multimodal
}

Video-Aided Analysis of Training in Synchronized Swimming:
A Multimodal Work Process

\author{
Dafne Muntanyola-Saura \\ Universitat Autònoma de Barcelona \\ www.academia.edu/dafnemuntanyola \\ Dafne.muntanyola@uab.cat (ESPAÑA)
}

Recibido: 01.042014

Aceptado: 18.12 .2014

\section{RESUMEN}

Este artículo propone una reflexión teórico-metodológica sobre una etnografía visual realizada en un entrenamiento deportivo. Planteamos un modelo integrado del conocimiento experto que explique las decisiones de las nadadoras y entrenadora del equipo olímpico español de natación sincronizada. Partimos de una recogida de datos etnográfica que integra la observación analógica, audiovisual y conversaciones con los participantes. El uso de herramientas del análisis cualitativo como el software ELAN muestra que la medición de la actividad social no debe seguir necesariamente modelos estadísticos. Es posible formalizar la interacción y la producción de significado social mediante un trabajo minucioso de trascripción y de sistematización de pautas visuales. Además, hacemos explícitos los principios arquitectónicos del software ELAN que configuran nuestra estrategia de codificación, evitando la reificación del proceso de entrenamiento en natación sincronizada. Nuestros resultados muestran la riqueza explicativa de esta metodología mixta de investigación en entornos deportivos, hasta ahora dominados por modelos estándar de investigación que no tienen en cuenta la naturaleza distribuida de las decisiones deportiva. 


\title{
PALABRAS CLAVE
}

Etnografía audiovisual, proceso de trabajo, reificación, historia natural, ELAN, conocimiento distribuido.

\begin{abstract}
This paper presents a theoretical and methodological discusssion on a visual etnography that took place in a sports training. We propose an integrated model of expert knowledge that explains the swimmers' and trainers decisión-making as part of the Spanish Olympic team. We Stara with an ethnographic data collection that integrates analogical observation, video observation and interviewsThe use of analytical tools such as ELAN software puts forward the possibility of measuring social activity not only with statistical models, but also by formalizing the interaction and the production of knowledge. We provide a detailed work of transcription and sistematization of visual patterns. Moreover, we make explicit the arquitectonic principles of ELAN software that build our codification strategy, avoiding the reification of the training process in synchronized swimming. Our results show the rich explanatory power of this mixed research methodology in sport environments, until now dominated by Standard models of research that do not take into account the distributed nature of decision-making in sports.
\end{abstract}

\section{KEY WORDS}

Audiovisual etnography, work process, reification, natural story, ELAN, distributed cognition.

\section{INTRODUCCIÓN}

Nuestro planteamiento de investigación es comprender cuáles son las pautas comunicativas que tienen lugar en los entrenamientos de natación sincronizada del equipo olímpico español. Desde un punto de vista metodológico, nos preguntamos hasta qué punto un análisis cualitativo de observaciones ethongráficas con herramientas audiovisuales puede arrojar luz sobre las pautas reales de interacción y comunicación entre la entrenadora y las nadadoras. Nuestro objetivo general consiste en operativizar y delimitar, aunque sea parcialmente, el proceso de trabajo de un entrenamiento de la natación sincro de un equipo olímpico.

Para saber lo que pasa en la cocina olímpica hemos optado por observar los entrenamientos y analizar su grabación en video con el programa de análisis cualitativo ELAN. La ciencia social que se alinea con el conductismo estricto responde que sólo podemos estudiar lo que está ahí fuera, es decir, lo que perci- 
bimos con nuestros sentidos: acciones, palabras, posturas, gestos y movimientos. En este artículo reivindicamos un término medio: creemos que la observación científica, incluida la etnográfica, siempre está mediada por lo percibido. Así pues en una etnografía audiovisual los investigadores deben decidir sobre el método(s) más adecuado para recoger y analizar la información recogida en vistas a conjugar analítica y compresivamente las dos instancias señaladas. El entrenamiento deportivo es necesariamente social al tener en cuenta la dimensión interactiva inscrita en toda actividad humana. Por lo tanto, la observación del entrenamiento en la piscina debe captar el proceso de trabajo que supone la natación de tal manera que informe suficientemente del conjunto de dichas instancias: los hechos tal y como suceden y los contextos en que se sitúan las implicaciones congnitivas y la pertinencia social. Finalmente, el entrenamiento olímpico es un entorno habitado y activado por profesionales expertos en su propio campo: la entrenadora por un lado y las nadadoras por otro.

Presentamos el proceso de entrada y de análisis de manera exhaustiva (Cicourel, 1974) para hacer explícitos todos los pasos de la investigación empírica, desde la entrada en el campo hasta la presentación de los resultados. Creemos que este nivel de detalle expositivo es necesario para captar la complejidad del proceso y así evitar los peligros metodológicos de la reificación y la intrusividad. Específicamente, en nuestro análisis aplicamos un ejemplo de análisis conversacional (AC) para ilustrar las propiedades comunicativas de los episodios de entrenamiento en la piscina. El AC nos da el marco más micro para explorar el carácter distribuido del conocimiento entre la entrenadora y las nadadoras.

La naturaleza de la grabación es de por sí un producto social de los agentes investigadores: un 'texto' que recoge una narrativa real. Ambos, imagen y texto, esperan ser descifrados en la investigación. La grabación no ha de contemplarse sólo como una expresión de la externalidad de los ensayos de la natación. Se ha de considerar también desde dentro, descubriendo el sentido con que los agentes implicados en los ensayos leen e interpretan los procesos que realizan. Tal perspectiva posibilita estar en consonancia con la idea de Bourdieu (2000:88) por la que las palabras - para nosotros también imagen y texto- se forman necesariamente en unas condiciones materiales, físicas y simbólicas -en nuestro lenguaje objetivadas y cognitivas- y además claramente bourdiano, es decir, cristalizadas en una determinada posición social- en nuestra perspectiva como un efecto de la apropiación desigual de los resultados de la interacción social. Así pues la imagen-texto (etnografía audiovisual) como expresión material, pero interpretado desde dentro de los ensayos y como resultados de interacciones sociales, nos da pistas para explicar las relaciones comunicativas y de poder que se producen entre los miembros del equipo en el proceso de creación de la coreografía que compitió en las Olimpiadas de Beijing.

La perspectiva adoptada como orientación para analizar los procesos de trabajo- y los ensayos lo son- conllevan otro supuesto y opción: la unidad de análisis no puede reducirse al individuo-agente sino que ha identificarse con el sistema de interacción en el que intervienen los dichos agentes, contextos situacional, mecanismos u otros. Por lo tanto, en nuestro análisis incluimos, primero, 
los recursos sociales de los agentes y de los instrumentos como expertos, luego la permanencia y reproducción de los atributos comunicativos, lingüísticos pero también más ampliamente gestuales, instrumentales y pragmáticos, que hacen de los participantes expertos en su entorno; y finalmente explicamos quién define la coreografía y cuál es el grado de reconocimiento de los participantes del entrenamiento.

\section{APROXIMACIÓN TEÓRICO-CONCEPTUAL}

La dimensión cognitiva que impregna el objeto de estudio es básica en los procesos interactivos y en consecuencia en los de trabajo. Estudios recientes de claro cariz cognitivo van en la dirección de la 'corporeidad' de la mente (o de la mente "corpórea") o con la expresión de conocimiento socialmente distribuido (Conein, 1994; Suchman, 1993; Clark, 2008 ; Gibbs , 2006 ; Hollan , Hutchins y Kirsh , 2000). Este enfoque tiene un gran valor añadido: explicando cómo se producen los mecanismos y procesos cognitivos (intencionales, representaciones, estrategias) y resultados en entornos locales o situacionales se desvela la caja negra de la psicología folk, y permite una explicación más integrada de la acción (Lozares, 2007; Muntanyola, 2014). La antropología cognitiva (D' Andrade, 1995; Hutchins, 2005) continúa la tradición durkheimiana de buscar una ontología de categorías cognitivas en los patrones relacionales y estructurales. En un marco weberiano, la acción es importante como parte de las tradiciones pragmáticas interactivos tales como la etnometodología (Garfinkel, 1967) la interacción simbólica (Goffman, 1961), la sociología cognitiva (Cicourel, 1974, 2012) y la etnografía (Knorr- Cetina, 1999; Wacquant, 2004, Lozares et al , 2013) .

La incidencia cognitiva es básica en los procesos interactivos puesto que interviene en las representaciones, objetivos, memoria, intencionalidades, etc. de los trabajadores, entre sí y con los artefactos. La cognición dentro de esta perspectiva no es un componente o instancia exclusivamente interna sino que se externaliza en los procesos de trabajo: en las pantallas, protocolos, conversación y resultados. De esta manera, y dada la unidad de referencia tomada, la cognición aparece además distribuida y puede así ser compartida como objeto de acuerdo (o desacuerdo) entre los agentes intervinientes. Por fin, se ha de tener también en consideración la importancia de la intervención de los artefactos en los procesos de trabajo. En este sentido, no se puede establecer una dicotomía entre acciones comunicativas, cognitivas y acciones instrumentales a partir de la distinción entre agentes humanos y los instrumentos de trabajo. Toda práctica interactiva proviene también y está mediatizada por los artefactos, máxime en los procesos de trabajo actuales: sistemas notacionales, automatización, mecanismos de información y cálculo, robotización, tecnologías cada vez más "inteligentes", que también están presentes en la preparación de los entrenamientos de natación sincronizada (Hutchins, 1995; Hutchins y Klausen, 1996; Lozares, 2001, 2007). 
Los procesos de trabajo han dado un vuelco radical desde el modelo representado por la cadena lineal a un modelo más complejo. No entramos en tal comparación sino en poner de manifiesto su naturaleza actual ya que los entrenamientos de natación sincronizada, como es el caso, es una clara ejemplificación de tal cambio y por tanto también de los procesos vinculados al deporte habitual o al de élite. Veremos pues cómo la perspectiva con que abordamos los entrenamientos de natación se acoplan a la adoptada sobre la naturaleza del proceso de trabajo. El proceso de trabajo, desde la opción que tomamos se define como (i) un conjunto de interacciones (de diferente naturaleza, fácticas, cognitivas...), (ii) entre agentes sociales (individuos o grupos) y/o artefactos tecnológicos, (iii) llevado a cabo en una situación espaciotemporal o entorno y en un contexto (desde un nivel más o menos limitado e inmediato hasta otros más genéricos macro y de diversa naturaleza) que interviene en el proceso (iv) en vistas a la modificación material o simbólica de una realidad fáctica y/o cognitiva y por tanto con un resultado o producto proveniente de la interacción. La componente social del proceso se genera en el hecho de que los resultados y/o recursos de la interacción (y por tanto del proceso de trabajo), y en todos los aspectos considerados, se distribuyen entre los agentes involucrados y además en todos los niveles en que se encuentren. Esta distribución puede se equitativa generando un 'nosotros' entre todos los agentes participantes o no equitativa originando los 'nosotros' y los 'otros' pe. ejemplo es el caso de los novicios y los expertos. En esta definición de los procesos de trabajo aparecen todas las instancias que se han venido considerando en las referencias conceptuales del objeto de estudio.

Tratando, en parte, de recoger la complejidad que concurre en un proceso de trabajo y los componentes e instancias que lo componen se señalan a continuación las líneas que orientan el diseño metodológico. Las líneas básicas de dicho modelo giran en torno al desarrollo del proceso de trabajo que supone los entrenamientos de gimnasia acuática.

(1) Primero, desde el punto de vista de la dinámica del proceso se consideran los aspectos siguientes:

(a) En primer lugar se da cuenta del entorno situacional. Kirsh (2001) lo interpreta como un 'espacio-momento-contenido' donde se realizan las interacciones y se generan las dinámicas fundamentales del proceso y donde, por supuesto, se hacen más manifiestos y 'a cuerpo entero' algunos de los agentes que intervienen, personas, ordenadores, comunidad de prácticas, grupo de trabajo, encargado... con sus recursos.

(b) En segundo lugar se considera el desarrollo de una o varias interacciones encadenadas de formas diversas cuyo sentido proviene de su articulación y dinámicas compartidas en el sentido de la producción de un resultado.

(c) Y por fin, la producción misma del resultado y de su grado de equilibrio: dicho resultado y su apropiación es lo que da identidad social al proceso pues establece unos estatus en función de tal nivel de apropiación. 
(2) La instancia cognitiva se plantea en el entrenamiento de natación sincronizada a partir de su naturaleza externalizada y contextual en los procesos de trabajo. En este sentido existen etnografías sensoriales recientes en los deportes que se centran en los corredores (Allen-Collinson y Hockey, 2013 ), en laboratorios (Knorr-Cetina, 1999, Myers, 2010), en hospitales (Muntanyola, 2010), mientras que en las artes existen estudios sobre el conocimiento experto de los músicos (Sudnow , 2009; Noya et al; 2010), los bailarines (Kirsh et al, 2009; Muntanyola y Kirsh, 2010) y los actores ( Noice y Noice 1997).

(3) La instancia cognitiva tiene su expresión como un resultado apropiado por los agentes, en procesos previos y en el actual examinado, bajo la expresión de conocimiento-agente experto vs. novicio. Esta dicotomía no puede considerarse como un atributo atomizado sino como una instancia, función y resultado de los procesos de trabajo. Siguiendo las consideraciones precedentes la distinción experto vs. novicio introduce pues una dimensión social: al mismo tiempo son, primero, agentes intervinientes en la interacción, segundo, resultado de la misma interacción y por fin dos modos y estatus de plasmación de los recursos cognitivos: experto vs. novicio como recursos específicos de dos agentes diferenciados. La identificación de dichos ambos agentes y tipo de conocimiento nos lleva a considerar:

(a) la sincronización de los participantes del entrenamiento en su entorno institucional

(b) el desarrollo temporal y espacial del proceso de instrucción y entrenamiento y

(c) el producto del entrenamiento, es decir en el caso que presentamos, la coreografía olímpica.

\section{METODOLOGÍA Y ANÁLISIS DE UN ENTRENAMIENTO DE NATACIÓN SINCRONIZADA}

El hecho de que se trate de una investigación etnográfica observacional no significa que no se pueda clasificar, tipificar y más genéricamente cuantificar. Aaron Cicourel, padre de la sociologia cognitiva, lo pone de manifiesto que la medición del proceso social en su totalidad requiere un estudio de la producción de sentido en la vida cotidiana (Cicourel, 1974). Sus entrevistas estructuradas y la estricta observación empírica de los entornos profesionales reales es un ejemplo de una investigación naturalista que se basa enteramente en métodos cualitativos de integración de múltiples niveles de análisis (Cicourel, 2007, 2012). Es posible realizar investigación audiovisual en deporte con una perspectiva metodológica que no sea estrictamente cuantitativa, basada en métodos estocásticos. La medición también se puede dar en un análisis como el que presentamos.

Proponemos un método naturalista basado en la observación con un tipo de 
análisis que sistematice los patrones de actividad de las interacciones locales entre los participantes de la formación deportiva. El líneas generales se siguen los principios de la grounded theory (Corbin \& Strauss, 1990) que mantiene el esquema de interpretación circular de los datos etnográficos y como método se utilizada el programa ELAN. Como innovación, hemos incorporado las técnicas de descripción jeffersonianas que provienen del método de análisis conversacional (AC) (ver apéndice). Se trata de una forma de transcripción que nos ha permitido entrar en el detalle de la interacción, no sólo a un nivel puramente lingüístico, sino a nivel multimodal, tomando en cuenta gestos, acciones corporales y otros elementos no verbales de comunicación.

Otro de los ingredientes metodológicos es el carácter multimodal del proceso de la interacción comunicativa en los entrenamientos. Siguiendo a Morana Alac (2005), el hecho de que se den sistemas de interacciones es, verbales y gestuales de carácter multimodal conlleva el uso de tecnología e instrumentos como la cámara de video y la pantalla de ordenador en el caso que nos ocupa, herramientas y los agentes. Alac (2005) llama a estos procesos socio-cognitivos de comunicación e interacción sistemas interactivos multimodales (SIM). Además, la pantalla de la filmación de los ejercicios de la piscina y la cámara de la entrenadora se convierten en anclajes materiales (material anchors) en Hutchins (2008) como medio de instrucción de la entrenadora y como material de un relato audio. En entornos expertos donde la tecnología es central, con en el caso de la natación sincronizada que nos ocupa, la manipulación de herramientas forma parte de los recursos multimodales existentes para iniciar la acción. Estos recursos están ordenados secuencialmente en el tiempo en una ajustada coordinación.

Las interacciones que tienen lugar alrededor de la cámara de vídeo y de la pantalla de la computadora pueden describirse en términos de toma de turnos conversacionales (turn-taking) . Partimos por lo tanto de las ideas básicas del AC sobre la toma de turnos como una actividad organizada, donde «en su gran mayoría, hay una sola persona que habla en un momento dado, mientras que el cambio de hablante se repite con la mínima separación y solapamiento (Have, 1999: 111). Del AC tomamos también su concepto de organización jerárquica de la interacción. En una conversación, lo habitual es que la palabra se tome de forma alternada entre los hablantes, y que el turno de uno termine cuando empiece el otro, que en términos de AC es "la selección del otro". Tomar la palabra sin que se esté respondiendo a el fin del turno de otro, que en $\mathrm{AC}$ se llama "auto-selección", es siempre una segunda opción, pero no la mejor. No obstante, la auto-selección es preferible a los monólogos interminables, es decir que en la vida cotidiana se prefiere el diálogo a la conversación monológica. En términos sociológicos, en una situación normal (dada por supuesta), la conversación respeta los turnos de palabra. La razón por la cual hablar sin parar es la última opción en la jerarquía de la interacción es precisamente porque existe una dinámica conversacional que emerge de esta cadena de turnos y que va más allá de la intención individual.

Schegloff (1996) enumera varias acciones que tienen lugar en la 
toma de turnos, como girar la cabeza hacia el futuro co-participante de la interacción, gesticular (Streeck y Hartge, 1992; Mondada, 2007), sonreir, aspirar o simplemente, hablar. Mondada (2009) señala también que la fase de apertura de los encuentros conversacionales se logra cuando los participantes metódicamente movilizan una serie de recursos posibles: trayectorias y posturas corporales, miradas, y todo el elenco de actos verbales, como palabras, gritos e interjecciones. Según el filósofo de la percepción Alva Noë (2004) la mirada, como experiencia, es una actividad de exploración que está mediada por nuestras habilidades sensorio-motrices. La mirada se convierte en un tipo de acción, como un gesto de las nadadoras o un movimiento en el agua. Noë, con una postura fenomenológica cercana a Hubert Dreyfus (1998), afirma: «Confiamos en que tenemos la habilidad de acceder a los detalles (de nuestra realidad física) con el movimiento, como base para nuestra sensación de presencia en el entorno como un todo (Noë : 2004 , 51). La mirada, entonces, no tiene solamente un carácter individual, sino también está relacionada con nuestro entorno físico, y nosotros añadimos, social.

Existen muchas contribuciones en sociología sobre lo audiovisual en el campo de la investigación, empezando por Bourdieu (1992), Becker (1974), Bancks (2001), Harper (2010) y más recientemente, el número especial sobre el análisis de vídeo de Qualitative Research (2010) y la recién fundada Journal of Video Ethnography, en la que uno de los autores participa como revisora. En esta revista se presenta al vídeo como un hecho sociológico. El video no sólo acompaña el texto, sino que es parte integral del material a revisar. Aún así, se publican pocas tesis sociológicas que trabajen con materiales multimodales como el que proponemos aquí, que se atrevan con la integración del texto o discurso verbal con la fotografía o el vídeo, que incluyan datos digitales, de audio o música, o bien objetos físicos o arquitectónicos (Muntanyola, 2009; Myers, 2012). Por otra parte, los diseños de investigación basados en imágenes y video como documentales o dibujos suelen identificarse con marcos teóricos específicos (Hesse-Biber, 2010; Knoblauch y Schnettler 2012) con nuevas maneras de explorar y comprender el mundo social a través del uso de lo o enfoques etnográficos y hasta experimentales, como el MA en Sociology del Goldsmith Collage de Londres. Por lo tanto, utilizar datos es no significa caer en una especie de iconografía ingenua (Mannay, 2010; Fele, 2012). Susan Sontag (1976) nos advierte en su clásico ensayo sobre fotografía cómo el hecho de tomar una fotografía implica un acto de seleccionar la información con lo que suponen un proyección de un conjunto de interpretaciones, valores y preferencias por parte de la investigadora. El proceso de investigación se ha desarrollado en tres pasos: el acceso al campo, la recogida de los datos empíricos (notas y video de campo) y el análisis y visualización de los resultados con el programa ELAN. 


\subsection{La entrada en el campo}

Aguiar y Schneider (2012) en la edición del libro "La investigación de las elites: desafíos y oportunidades en estudiando hacia arriba" presentan el concepto de estudiando hacia arriba (Studying Up). Se refieren a cómo los investigadores de clase media-alta con elevado capital cultural tienen un acceso más fácil a los grupos sociales con mayor estatus socioeconómico. De acuerdo con el autor y siguiendo el clásico trabajo sobre el tema de la élite del poder, de Wright Mills (1956), una manera de estudiar las élites es mediante la observación y la entrevista a aquellos individuos cuyas decisiones tienen una amplia influencia sobre el bienestar económico y financiero en los estados y sobre los individuos. La definición de Aguiar del los grupos de élite se amplía e incluye a los artistas, famosos y deportistas importantes con altos privilegios de reconocimiento y de estatus socio- cultural (Hondagneu-Sotelo, 2010). Dado que la introducción en el campo de estudio exige un proceso de negociación, pues significa una especie de invasión y apropiación de otro dominio, con el "guardián" (el gatekeeper del entorno a estudiar); es un tema clave al proponer una etnografía. Es pues importante, en la negociación, comenzar por explicar en este proceso de entrada y de acceso a la información, el contenido, objetivos y beneficios, incluso mutuos, de la investigación. La misma negociación puede ser parte del proceso de observación. Por otro lado, Ostrander (1993) en su artículo clásico sobre el acceso y la construcción de relaciones con las élites, afirma la necesidad de ser directo en el trato con los participantes del entorno observado.

Nuestra etnografía de natación sincronizada se inició en 2012. La estrella del equipo olímpico español estudiaba en la Universitat Autònoma de Barcelona ( UAB) y contactó con uno de los directores del proyecto, desde el departamento de filosofía en la UAB. En una primera conversación informal, coincidieron en la idea de que observar a las nadadoras y explorar proceso creativo del entrenamiento a través de la observación, la filmación y entrevistas sería una forma interesante de explorar cómo los atletas piensan y toman decisiones. La negociación definitiva de entrada se inició en el Centro de Alto Rendimiento (CAR) del equipo. Tanto la entrenadora como las nadadoras demostraron sensibles a nuestra petición. Además la negociación se planteó como un intercambio de beneficios mutuos. Aún así, pudimos experimentar la existencia de 'fuertes guardianes' (gatekeepers) entre los directores del CAR que nos pidieron seguir un horario rígido de acceso a la piscina, previos avisos y un acuerdo con el centro y no solamente con la entrenadora y las nadadoras, lo que hizo que nuestras observaciones fueran a veces complicadas y un tanto difíciles. El equipo español de sincronización es uno de los mejores equipos olímpicos y como tal pertenece claramente a una elite social; su protección y aislamiento de la sociedad en su conjunto, en tanto que élite deportiva, es un hecho.

Las observaciones se llevaron a cabo en la primavera de 2012, con 2 cámaras de vídeo. Tanto la entrenadora como las nadadoras participaron en la observación y las entrevistas. No hubo ninguna brecha jerárquica entre los investigadores y las investigadas. Otro elemento que indica el grado de confianza 
fue la accesibilidad a los números de móvil de y entre los/as investigadores/ as, entrenadora y nadadoras. Desde el punto de vista de género, se trata de un deporte exclusivamente femenino: entrenadora, su auxiliar y las nadadoras. Sin embargo, otros agentes mas contextuales presentes en la piscina de forma intermitente, como el fisioterapeuta, el técnico postural y de movimiento y algún miembro del jurado eran hombres.

\subsection{Los instrumentos de recogida de la información}

El uso de herramientas audiovisuales en la recogida de datos abre la posibilidad a la reificación si se cae en el supuesto de que «todo está allí». La reificación define la tendencia a sobreestimar la capacidad de procesar la información (Pylyshyn, 2003). Los científicos sociales caen fácilmente en la falacia escolástica de poner un tipo de acontecimiento (pe. el nivel de rendimiento estándar o deseado de una nadadora) en lugar del observado (la actuación de la nadadora en un entrenamiento real). Siempre existe el riesgo de perder aspectos importantes o suponer otros en la interpretación. La virtud de la literalidad de la cámara y la aparente transparencia de las imágenes de video tienden a ocultar el carácter selectivo de la filmación. Algunas acciones o decisiones importantes pueden caer fuera del campo de la cámara o simplemente pueden tomarse fuera del entorno de la piscina observada en reuniones previas o posteriores entre miembros del equipo. Por el contrario, acciones irrelevantes para el estudio, como conversaciones privadas por el móvil de la entrenadora o momentos en los que las nadadoras se están cambiando, pueden ocupar demasiado espacio en la filmación. Uno tiene que saber dónde poner la cámara para capturar los patrones de comunicación pertinentes para el estudio sociológico. El estudio debe tomar decisiones estratégicas a partir de notas de campo exploratorias que definen los lugares y objetivos del proceso de entrenamiento.

Otro peligro metodológico que es frecuentemente citado al hablar del uso de herramientas audiovisuales es la intrusividad. Para una reflexión más detallada sobre las consecuencias metodológicas del uso del video en la observación, ver el artículo de la misma autora publicado también por empíria sobre el trabajo en equipo de una unidad médica (Muntanyola, 2010). Tres elementos entran en juego en la observación etnográfica: la imagen del objeto (lo que percibimos y oímos en la observación), el medio a través del cual el observador se fija en el entorno (la mirada, el visor) y, por último, la perspectiva sociológica que se construye teóricamente . Además, en la piscina la tecnología está muy presente, como se ve en la figura 1. Las nadadoras olímpicas se ven a sí mismas continuamente en la pantalla, en la que la entrenadora proyecta su actuación filmada en video in situ. La cámara, por lo tanto, es una ancla material (Hutchins, 2005) para la entrenadora y las nadadoras y también es parte de proceso de trabajo en el día a día. Las nadadoras tienen cámaras por debajo y por encima del agua: la entrenadora utiliza los mandos de reproducción y de 
rebobinado de la cámara para mostrar inmediatamente a las nadadoras lo que ha ido mal y así instruir y corregir la coreografía olímpica.

Figura 1. La cámara de video y la pantalla como anclas cognitivas, Barcelona, 2013.

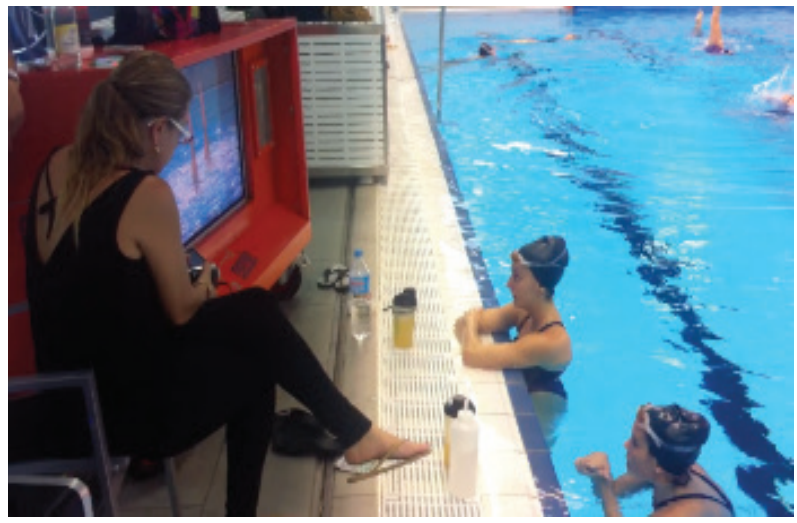

La observación incorpora necesariamente interacciones imprevistas con los miembros del equipo observado. Por ejemplo, durante el trabajo de campo la entrenadora interpeló en el proceso de formación a la investigadora: mientras la entrenadora daba instrucciones a las nadadoras les preguntó si conocían la historia de Lot en el Antiguo Testamento. Al desconocerlo, la entrenadora pidió a la investigadora que lo explicara. Este tipo de interacción, a parte de ser una interesante muestra del uso de la metáfora en la instrucción deportiva (porque el objetivo de la historia era que las nadadoras giraran la cabeza como lo hace Edith antes de convertirse en una estatua de sal) muestra que la entrenadora era bien consciente de la presencia de la investigadora en la piscina. En este ejemplo la entrenadora no considera que la cámara sea un obstáculo para el diálogo pues es percibida como una extensión del ojo de la investigadora. La presencia de la observadora debe ser negociada como en cualquier contexto etnográfico. Pueden producirse dinámicas de interés que hacen explícitas contribuciones relevantes al desarrollo de la investigación.

\subsection{La elección del programa de análisis y la interpretación del material etnográfico.}

Llevada a cabo una primera observación, la estrategia de análisis consistió en realizar una primera codificación, primero en Excel y después en ELAN, para obtener indicadores más específicos de observación. Los códigos se 
construyeron a través de lecturas, discusiones y conversaciones informales con los participantes (Strauss \& Corbin, 1990). Más específicamente, el diseño de la codificación fue posible porque un modelo teórico basado en la cognición distribuida guió el proceso de análisis (Hollan et al, 2000, Hutchins, 2005, Kirsh el al, 2009). Como hemos indicado anteriormente, la horizontalidad del proceso de entrada facilitó la naturalidad de las conversaciones.

El uso del vídeo multiplica el volumen de información recopilada. Varios programas de análisis, actualmente disponibles en el mercado, satisfacen las necesidades de un científico social que busque herramientas para organizar, analizar y presentar datos cualitativos. El programa ELAN fue desarrollado originalmente por el Instituto Max Planck de Psicolinguiística para el análisis de micro- gestos e interacciones (Brugman y Hussel , 2004). El entorno web del porgrama ELAN es un foro abierto donde los usuarios intercambian información transversal, formando una red de distribución de recursos cognitivos más cercanos a las necesidades empíricas de la investigación.

Estas reflexiones metodológicas nos llevan plantear la relación entre la creatividad del investigador y las limitaciones de los programas de análisis. Tanto los opositores como los defensores de los programas pueden caen en un error epistemológico: atribuyen causalidad a un software de análisis particular sin tener en cuenta que raramente se utilizan de forma aislada. Los investigadores utilizan ELAN, Transana o Atlas.ti, por nombrar algunos de los software cualitativos más conocidos, en combinación con otros que llamamos programas auxiliares. Los programas auxiliares son versátiles, suelen ser gratuItos o de menor precio que los centrales (aunque ELAN, de momento, es gratuito) y se pueden aplicar tanto a datos verbales (Excel, Word, Power Point) como audiovisuales (Quicktime). En la etnografía de natación sincronizada utilizamos ELAN como software central (figura 2), mientras que Word, Excel y Quicktime quedan como auxiliares. Las notas de campo tomadas en Excel durante el proceso de entrenamiento ayudaron en la transcripción de las observaciones comunicativos. A diferencia de Atlas.ti o InVivo, ELAN favorece la transcripción y la codificación simultánea de diversos aspectos del proceso, incluyendo el vídeo completo sin fragmentación lo que facilita la clasificación de las interacciones y la introducción de las-transcripciones de las narrativas 
Figura 2. Transcripción y análisis cualitativo con el programa ELAN.

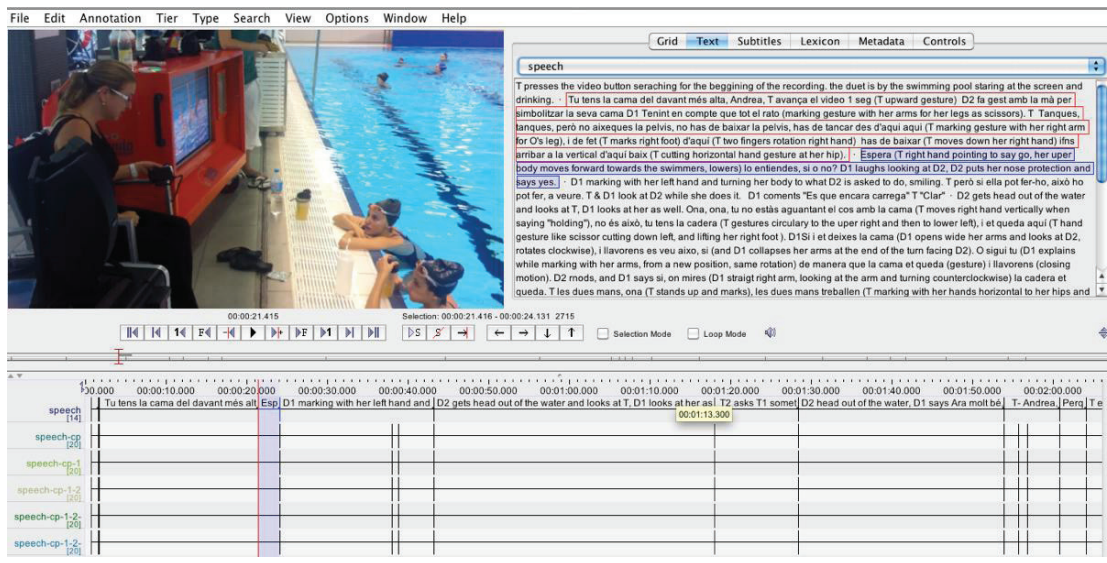

La unidades significativas para el análisis son situaciones interactivas recurrentes o, en otros términos, episodios de actividad recurrente (Activity Recurrent Episodes o ARE). Estas unidades son relevantes en un contexto de aprendizaje. Su inicio y acabamiento se producen desde luego cuando se dan cambios en el contenido o naturaleza de la interacción-(Barab, Yamagata Hay y Lynch, 2001: 66). Otros criterios que dan identidad a la unidad son la introducción de nuevos agentes y/o su desaparición, a nuevos escenarios contextuales y sobre todo a una cierta complitud de los resultados con un cierto grado de estabilidad. Se observaron pues las interacciones entre las entrenadoras y las nadadoras en la piscina olímpica. Los agentes son considerados como expertos en sus diferentes ámbitos dentro de un tipo eminentemente competitivo con respecto a otros equipos; se trata de las olimpiadas.

Una primera exigencia consiste en determinar los elementos, ingredientes, agentes, contextos, dinámica, etc., de las unidades significativas. Es una exigencia para la codificación sistemática de la información recogida (Corbin y Strauss, 1990). En este sentido definimos y determinamos un proceso de entrenamiento que incluye los participantes, las herramientas o artefactos, las interacciones y/o acciones, la ubicación en contextos y el contenido general de la interacción comunicativa del entrenamiento. Construimos un proceso estándar de entrenamiento puesto que el objetivo consiste en analizar e interpretar la interacción y como se configura y desarrolla el proceso para llegar a un estado satisfactorio del entrenamiento que corresponde a la unidad significativa de interacción analizada y con ello el resultado de la creatividad de las nadadoras y la entrenadora (Figura 3). En este sentido hemos dividido el proceso de de trabajo formativo estándar en siete fases, separadas por estados de transición. Cada fase se refiere al conjunto de interacciones que corresponden a la realización de una sección completa de la coreografía.

Una vez completada esta fase se exportan a Excel los contenidos que 
corresponden a cada una de las interacciones; de esta manera se obtiene el cómputo de sus modalidades diferentes. Este análisis preliminar pone de manifiesto cómo las instrucciones verbales se combinan con la manipulación constante de la cámara de vídeo y la visualización de la propia actuación en la pantalla. La figura 3 muestra cómo el 100\% del tiempo de ensayo incluye las interacciones entre el entrenador y las nadadoras ( $83 \%$ si no consideramos la mirada como un medio para la interacción) y, de éstos, el $81 \%$ incluye la comunicación verbal (siguiendo Lunardi y Lansagan en Muntanyola, 2014). El habla y las herramientas a menudo se superponen.

Figura 3. Proceso de entrenamiento estandarizado y estadísticas exploratorias de multimodalidad

\begin{tabular}{|c|c|c|c|c|c|c|c|c|c|}
\hline $\mathrm{O}^{\prime}$ & & & & & & & & \multirow{2}{*}{\multicolumn{2}{|c|}{$T 3 b$}} \\
\hline $\begin{array}{c}\text { Interactive } \\
\text { Phases }\end{array}$ & Prep & p1 & $T 1$ & P2 & $T 2$ & P3 & $T 3 a$ & & \\
\hline Speech & $\mathrm{s}$ & & $\mathrm{s}$ & & $\mathrm{S}$ & & $\mathrm{s}$ & \multicolumn{2}{|c|}{$\mathrm{s}$} \\
\hline Tools & $T$ & $T$ & & & $\mathrm{~T}$ & & & \multicolumn{2}{|c|}{$T$} \\
\hline Duration & 1,1 & 0,3 & 1,7 & 0,8 & 0,3 & 0,8 & 0,3 & \multicolumn{2}{|c|}{6,1} \\
\hline$\%$ & 3,98 & 1,16 & 6,09 & 2,89 & 1,1 & 2,71 & 1 & \multicolumn{2}{|c|}{21,39} \\
\hline & & & & & & & & & $28^{\prime}$ \\
\hline \multirow[t]{3}{*}{ T3c } & P4 & \multicolumn{3}{|c|}{$T 4 a$} & P5 & T5a & p6 & P7 & Coda \\
\hline & & \multicolumn{3}{|c|}{$\mathrm{s}$} & $\mathrm{S}$ & $\mathrm{s}$ & & & $\mathrm{S}$ \\
\hline & & \multicolumn{3}{|c|}{$\mathrm{T}$} & & & & & $\mathrm{T}$ \\
\hline 3,8 & 1 & \multicolumn{3}{|c|}{8,3} & 0,9 & 0,8 & 0,3 & 0,7 & 1,2 \\
\hline 13,51 & 3,59 & \multicolumn{3}{|c|}{29,17} & 2,99 & 2,96 & 1 & 2,57 & 4,05 \\
\hline
\end{tabular}

La intención de estos tipos de análisis e interpretación consiste en 'reproducir' la complejidad de los procesos de trabajo, al menos en los componentes y niveles claves. Dichos tipos de codificación permiten diversas lecturas de los procesos según los intereses y objetivo de la investigación: la trayectoria y su dinámica, las modulaciones, los contenidos, las apropiaciones, etc. Con el fin de identificar y analizar la dinámica de la interacción en la lógica del análisis conversacional se selecciona y transcribe un fragmento de la descripción narrativa (Figura 4). Este fragmento se considera en la fase específica como la unidad más pequeña de la interacción significativa (o Actividad recurrente Episodios (ARE) Elegimos fase la T4a del proceso debido a su céntrica ubicación, duración y la presencia tanto en el discurso y artefactos como vehículos de comunicación. 
Figura 4. Descripción del Episodio Recurrente de Actividad (ARE) seleccionado para el análisis de video.

\begin{tabular}{|l|l|}
\hline & Episodio Recurrente de Actividad \\
\hline Inicio & $\mathbf{2 4}$ min; 40 seg. \\
\hline Entrenadora-dueto & $\begin{array}{l}\text { Dueto (S1y S2), después de practicar unos pasos de la } \\
\text { coreografía, nada hacia el lado de la piscina, miran la pantalla y } \\
\text { la entrenadora (T) les da instrucciones para corregir una cadera } \\
\text { mal puesta de una de las integrantes del dueto (S2). }\end{array}$ \\
\hline Herramientas & $\begin{array}{l}\text { T para música, pasa las imágenes de la cámara de vídeo } \\
\text { hacia delante y hacia detrás, habla S1 y S2, S1 y S2 hablan } \\
\text { entre ellas, S1 explica con marcajes a S2 lo que ha hecho mal, } \\
\text { traduciendo en otra modalidad lo que les ha dicho T. El dueto } \\
\text { vuelve al centro de la piscina y repite la coreografía, T a gritos } \\
\text { las corrige. }\end{array}$ \\
\hline Fase de actividad & Transición 4a \\
\hline Final & 33 min. \\
\hline
\end{tabular}

En la figura 5 se muestra una instantánea de 4a ARE ya transcrita en ELAN. El análisis se exporta a continuación a Word, a fin de transcribir, con detalle 'jeffersoniano' los niveles de interacción y comunicación. Las tomas instantáneas pasan también a ELAN para completar la transcripción y dar cuenta de todas las modalidades de comunicación. En ello se sigue el modelo secuencial de Schegloff y Sacks (1973) así como las convenciones de Jefferson presentadas por Duranti (1997), Mondada(2008), Goodwin (2013) y Heath \& Luff (2013). 


\section{Figura 5. Transcripción detallada del ARE con ELAN.}

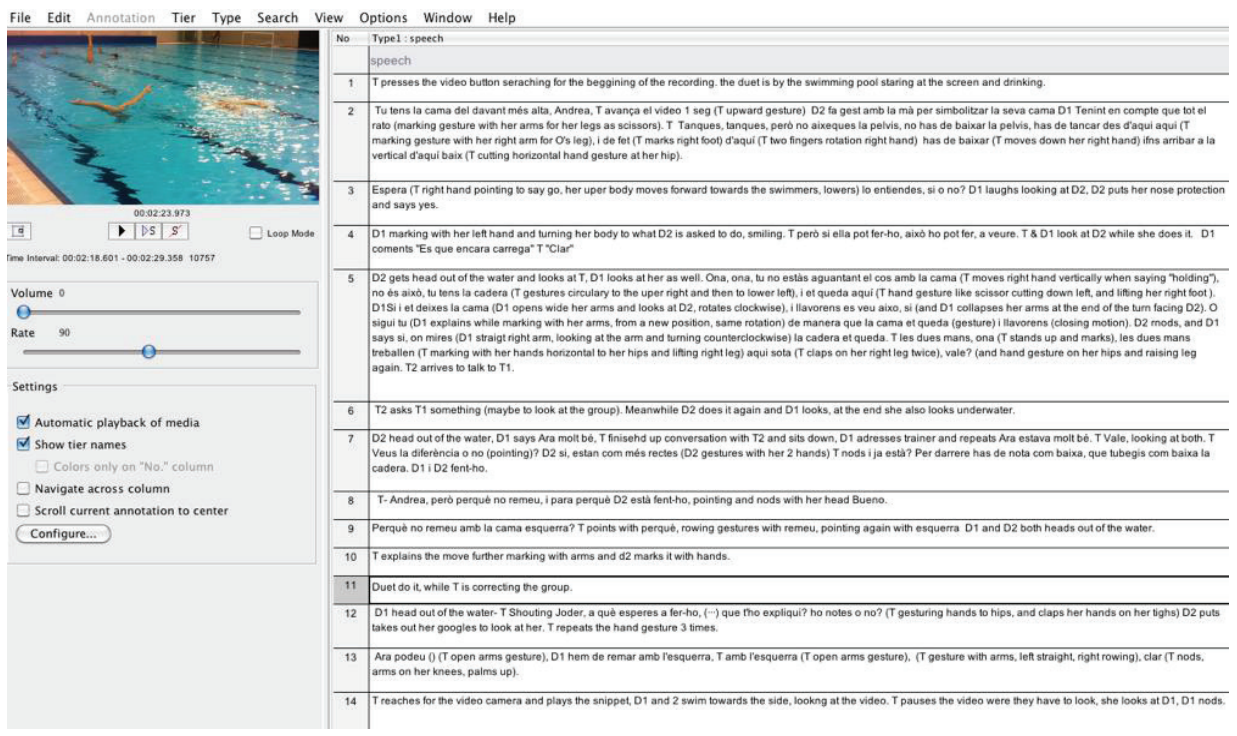

El análisis detallado que ofrecemos en la figura 6 que corresponde a un momento de interacción muestra como las instrucciones verbales se combinan con el gesto, el marcaje (Kirsh y Muntanyola, 2010) y la manipulación constante de la cámara de video como ancla material (Hutchins, 2005). La entrenadora corrige a la nadadora S2, una de las dos nadadoras del dúo que no levanta tanto la cadera cómo la otra nadadora del dueto, S1, en el movimiento coreográfico específico de la pantalla. La dinámica que vemos en la figura 7 constituye lo que Morana Alac (2005) llama un sistema multimodal interactivo; es decir, un conjunto de interacciones visuales, verbales y gestuales, junto con las herramientas y los agentes. Este sistema socio-técnico es la base para los entrenamientos olímpicos que se desarrolla alrededor de la pantalla y el discurso de la entrenadora.

Los cambios de turno de la entrenadora y las nadadoras en la figura 6 informan acerca de la complementariedad de las correcciones proporcionadas por S1 y la entrenadora dentro del marco interactivo de la fase analizada. En primer lugar, la entrenadora sigue un intercambio entre S1 y S2, donde S1 añade la valiosa información de un truco: una ayuda visual para que S2 mantenga la cadera en el lugar correcto (07). La entrenadora espera que S1 termine su marcaje con las manos, su cuerpo orientado en línea con S2 (\#1) para pedir verbalmente a la confirmación de S2 (08). Deja que S1 recupere la conversación (09). Su expresión verbal se superpone con la entrenadora y continua con el marcaje, dando una segunda aclaración informativa a S2 , esta vez no visual, sino en relación con el empuje del movimiento de la pierna. La mirada de S2 indica que su atención pasa a S1. En el siguiente turno de palabra, los gritos de 
la entrenadora interrumpen, S1 (10); al tomar la palabra (se auto-selecciona) grita más instrucciones. Este tercer elemento de información se suma a las correcciones anteriores de S1. La entrenadora enfatiza con el tono de voz, la repetición, interpelando a la nadadora S2 por su nombre y marcajes (marking) (\#2). El marcaje es una estrategia cognitiva común a bailarinas y nadadoras, así como a los músicos y otros artistas que trabajan con el cuerpo (Muntanyola y Kirsh, 2010). Permite la comunicación sin hacer el movimiento o la acción completa, mediante la selección de aspectos tales como el peso, la velocidad, la dirección o la dinámica. Estos son los elementos del paso "equivocado" que S1 y la entrenadora están explicando a S2 para corregir una cadera demasiado baja.

En este fragmento, por lo tanto, la nadadora S1 completa y al mismo tiempo selecciona la información dada por la entrenadora: ella es a la vez la extensión, al marcar con más detalle y con mas información relativa a la orientación del cuerpo y a la mirada lo que la entrenadora está diciendo, y la intérprete, como cuando filtra la información relevante proveniente de la actuación de la otra nadadora S2. Su competencia como nadadora sincronizada, que es lo que le falta a la entrenadora que ya no compite, se encuentra en el uso experto de su propio cuerpo como una herramienta de comunicación bajo el agua.

Figura 6. Fragmento de ARE (61-73s) con un análisis conversacional multimodal

7. S1 Si, on mires Yes, where you look hand gesture with open hand, then straight arm \#1 la cadera et queda. the hip remains. Looking at her arm

*Turning counterclockwise, straight right arm* T looks at S2 when S1 is finished with her turn

8. T Vale? Ok?

9. S1 [Si Yes]

S2 looks back at $\mathrm{T}$

S1 que el cop del peu [t'impulsi] The foot's kick should [push you]. *Marking leg with right arm*

$\mathrm{T}$ and $\mathrm{S} 2$ gazing at $\mathrm{S} 1$

10. T [LES DUES MANS] [BOTH HANDS]

S2!

$+\mathrm{T}$ leaves cam on the floor and stands up+

$\underline{S 1}$ gaze towards the screen \& S2 gaze towards T

T Les dues mans Both hands

+Marking opens her hands horizontal to her hips+

T gaze on her hands, $\mathrm{S} 1$ and $\mathrm{S} 2$ gaze towards the $\mathrm{T}$

\#2 $\mathrm{T}$ treballen aqui sota work down here

+Marking hands twice horizontal to her hips and lifting right leg+ $\underline{\mathrm{T} \text { looking at } \mathrm{S} 2}$ 


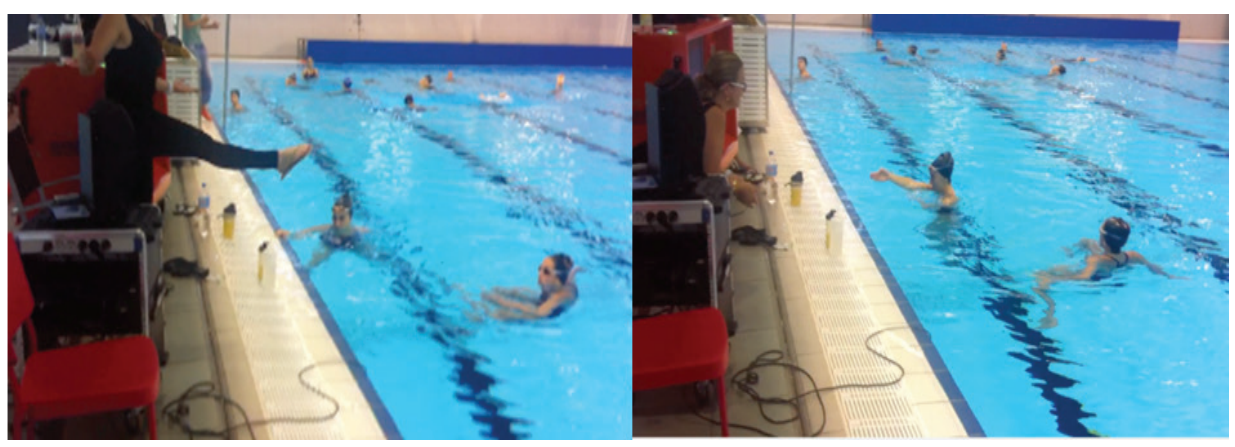

$\# 1$

\#2

El análisis micro del proceso nos permite tomar en cuenta (a) la sincronización de los participantes del entrenamiento en su entorno institucional (b) el desarrollo temporal y espacial del proceso de instrucción y entrenamiento y (c) el producto del entrenamiento, es decir en el caso que presentamos, la coreografía olímpica. En nuestro análisis incluimos primero los atributos, los recursos sociales de los agentes y de los instrumentos como expertos, luego la permanencia y reproducción de los atributos comunicativos, lingüísticos pero también más ampliamente gestuales, instrumentales y pragmáticos, que hacen de los participantes expertos en su entorno; y finalmente explicamos quién define la coreografía y cuál es el grado de reconocimiento de los participantes del entrenamiento.

Retomando la dimensión del conocimiento experto que introducíamos más arriba, nuestro análisis ha puesto de manifiesto los momentos de sincronización de los participantes a partir de los cambios de turno en el entrenamiento. Además, la composición multimodal y altamente interactiva de los ARE de instrucción entre la entrenadora y las dos nadadoras muestra como se trata de un proceso de trabajo y cognitivo localizado en el espacio y en el tiempo, claramente intersubjetivo y, por lo tanto, contextualizado. Finalmente, el producto del entrenamiento, la coreografía olímpica, es el resultado emergente de este proceso de interacción. Si bien la autoridad (y autoría) recae sobre la entrenadora, vemos en momentos del proceso, como en el fragmento analizado en la figura 6, cómo existen momentos de toma de decisión distribuidas, es decir, donde la información es interpretada, filtrada e incluso formulada a partir de la iniciativa de las nadadoras, y no sólo desde la entrenadora. Esta flexibilidad en el grado de intervención de los participantes, de sus funciones y responsabilidades en el entrenamiento, muestran la naturaleza dinámica y distribuida del conocimiento experto en deporte. 


\section{CONCLUSIONES}

La etnografía identifica, analiza e interpreta las interacciones sociales de los agentes y sus artefactos en vistas a descubrir su sentido y significado. La etnografía audiovisual como método tiene el mismo objetivo: posibilita al sociólogo al hacer emerger los significados a partir de la observación y recopilación de datos y en su caso también relacionarlos con su marco teórico. Las imágenes que se toman fuera de contexto, es decir sin texto, caen en la cautividad de los estereotipos. En la ciencia, tal demanda se traduce en la necesidad de no tomar las imágenes del video por sentado así como tampoco la aplicación de esquemas de codificación reflexiva y teóricas de los datos recogidos. La enorme cantidad y variedad de datos que incorpora la etnografía de vídeo puede ser abrumador para el investigador.

Combinamos el uso del programa ELAN con los programas auxiliares Word y Excel. Hemos trabajado con los modelos cognitivos de Lozares $(2000,2001)$, Alac (2005) y Kirsh (2007), con los correspondientes conceptos de proceso de trabajo, sistema interactivo multimodal y marcaje. Estos conceptos nos han permitido analizar en detalle los procesos de interacción y de comunicación que están en la base de todo entrenamiento deportivo. Como estudio de caso, hemos presentado un trabajo etnográfico del equipo de natación sincronizada español.

Gracias a este trabajo de observación y de análisis audiovisual, hemos podido definir, dentro del proceso de trabajo, fenómenos empíricos tales como gestos de la entrenadora, herramientas cognitivas de las nadadoras como la cámara o la pantalla, y estrategias del uso experto del cuerpo como el marcaje (Muntanyola y Kirsh, 2010). Los fenómenos detallados gracias al análisis conversacional ponen de manifiesto otras dimensiones del proceso de entrenamiento complejos, tales como la necesaria orientación multimodal del entrenamiento deportivo, la existencia de anclajes materiales (Hutchins, 2006) para las instrucciones de la entrenadora, o la existencia de estructuras de filtrado, en las que las nadadoras se ayudan entre ellas para responder a las instrucciones de la entrenadora.

Mientras que la entrenadora da instrucciones multimodales (a través del habla, el marcaje y el gesto), la nadadora más experta (S1) da a su compañera (S2) explicaciones informativas y de carácter evaluativo a través del marcaje $\mathrm{y}$ con un discurso detallado que filtra y mejora la comprensión de lo que quiere la entrenadora por parte de la nadadora. Por tanto, este nivel de análisis plantea la naturaleza distribuida de la atención, ya que un tercer participante, la nadadora con mayor experiencia en el dúo, actúa como los ojos y el cuerpo de la entrenadora.

La observación etnográfica y analizada por ELAN revela la especificidad de la información transportada por cada modalidad, además de la complementariedad entre los diferentes tipos de interacción. La captura de vídeo ha permitido preservar los momentos en los que los bailarines / nadadores trabajaron juntos. El experto no puede separarse de actor social y por lo tanto su rendimiento interactúa con ambos modelos de comunicación 
y confianza, y configura sus tomas de decisión (Goffman, 1961; Mondada, 2012). Estas interacciones narrativas y técnicas, como las transcritas en nuestra transcripción, se vinculan a los patrones de confianza que existen en entornos profesionales. La importancia de la confianza comunicativa en los pasillos y en los momentos de instrucción recuerda el concepto de chismorreo técnico (technical gossip) (Knorr-Cetina, 1999). Knorr-Cetina aplica este término a las decisiones que se toman alrededor de la máquina de café en los laboratorios. En la piscina, las decisiones se toman in situ, en el momento, pero adoptan también pautas informales y multimodales parecidas. Futuros trabajos en esta línea de investigación incluirán también más entrevistas semi-estructuradas a las nadadoras y entrenadora para comprender mejor la perspectiva subjectiva del proceso de trabajo.

El uso de las herramientas digitales necesita una mayor flexibilidad metodológica y un mayor conocimiento de la arquitectura del programa de análisis. Los que afirman que las herramientas audiovisuales son demasiado intrusivas y que pueden perturbar la normalidad de los ensayos o entrenamientos también creen que el uso de un programa de análisis cualitativo como ELAN limita la imaginación sociológica. Lo que llamamos principios incluyen los elementos que hemos especificado en nuestra historia natural en comparación: la necesidad de codificación teórica de evitar la reificación, la relevancia del proceso de entrada, la función reflexiva del investigador y la cámara como agentes potenciales de intrusión, la pluralidad de el software utilizado en el campo, y la captura de vídeo virtudes de grabación y múltiples ángulos que conserva la integridad del proceso de formación. Todos estos principios de la arquitectura del proceso de investigación dan forma al uso de herramientas y la mejor estrategia de codificación en la etnografía visual, así como en otros métodos cualitativos. La literalidad favorece el análisis de la interacción y la multimodalidad. Pero es gracias a la codificación teórica y un análisis reflexivo que se evita la reificación en procesos de trabajo complejos, como el entrenamiento deportivo que aquí presentamos.

\section{Apéndice 1: Convenciones para la transcripción}

Se presenta una transcripción literal por línea, su objetivo es ayudar a la lectura del texto original.
talk
talk habla original
[] WHAT?
traducción aproximada
(()) habla superpuesta
() voz alta
(2) habla que no se oye bien

Los detalles multimodales se han transcrito siguiendo las convenciones siguientes: las acciones (incluyendo gestos y marcaje) se decriben a 
continuación y se han sincronizado siguiendo el formato siguiente

** Describe las acciones de un participante (S1)

**** Describe las acciones de otro participante (S2)

$++\quad$ Describe las acciones de un tercer participante (T)

Gaze dirección de la mirada

\#12 momento en el que se ha tomado una imagen de la acción.

\section{BIBLIOGRAFÍA}

AGUIAR, L \& SCHNEIDER, C. (Eds.) (2012) Researching Amongst Elites: Challenges and Opportunities in Studying Up. London: Ashgate.

ALAC, M. (2005) From Trash to Treasure: Learning About Through Multimodality Brain Images. Semiotica, 156-1/4, pp.177-202.

BANKS, M. (2005) Visual Methods in Social Research. London, Sage.

BARAB, S., HAY, K. AND YAMAGATA-LYNCH, L. (2001) Constructing Networks of Action-Relevant Episodes: An In Situ Mehodology Research, The Journal of the Learning Sciences, 10, pp. 63-112.

BASSETTI, C. (2013). Male dancing body, stigma and normalizing processes, Recherches sociologiques et anthropologiques, 2: 67-90.

BELTING, H. (2004) anthroplogie Pour une des images. Paris, Gallimard.

BOURDIEU. P. AND WACQUANT, L. (1992) Per reflexive sociology. Barcelona: Herder.

BRUGMAN, H. \& HUSSEL, A. (2004). Annotating Multimedia/Multimodal Resources with ELAN. Proceedings of LREC 2004. 4th International Conference on Language Resources and Evaluation.

CICOUREL, A. V. (1974) Cognitive Sociology. New York, The Free Press.

CICOUREL, A. V. (2006) Cogntive / Affective Processes, Social Interaction, Social Structure and Representational Re-descriptions as: their contrastive bandwidths and spatio-temporal foci, Mind and Society. 5, pp. 39-70.

CICOUREL, A. V. (2012). Origin and Demise of Socio-cultural Presentations of Self from Birth to Death: Caregiver 'Scaffolding' Practices Necessary for Guiding and Sustaining Communal Social Structure Throughout the Life Cycle, Sociology, February 2013; vol. 47, 1: 51-73.

CONEIN B. Y JACOPIN E. (1994). "Action Située et cognition, le savoir en place", Sociologie du Travail, 4, 475-500

D'ANDRADE, R. (1995) The Development of Cognitive Anthropology, Cambridge University Press, Cambridge.

FERRAND, M., IMBERT, F. AND MARRY, C. (1999) Excellence Scolaire: Une affaire de famille. Le cas des normaliennes et normaliens scientifiques. Paris: L'Harmattan.

SCIENCE, CAMBRIDGE, CAMBRIDGE UNIVERSITY PRESS. GARFINKEL, H. (1967) Studies in Ethnometodology, Los Angeles, Polity Press.

GIBBS, R.W. (2006) Embodiment and Cognitive Science, New York, Cambridge University Press.

GLON, M. (2007) "La Matérialité de la théorie". Conference Paper in Colloque international de recherche en danse, Centre National de la Danse, 21-24 June, Paris. 
GOFFMAN, E. (1961) Asylums, New York, Anchor Books.

HOLLAN, J., HUTCHINS, E. AND KIRSH, D. (2000) Distributed Cognition: Towards a New Foundation for Human-Computer Interaction Research. ACM Transactions on Computer-Human Interaction, 7, 2: 174-196.

KIRSH, D. (2007)- El uso del espacio (The Use of Space) in Lozares, C. (ed.) Interacción, redes sociales y ciencias cognitivas. Granada: Comares.

KIRSH, D. (2012). Running it through the body. Proceedings of the 34th Annual Cognitive Science Society. Lawrence Erlbaum.

KIRSH, D. AND MUNTANYOLA, D., LEW, A., JAO, J. AND SUGIHARA, M. (2009) Choreographic methods for creating novel, high quality dance, Design and Semantics of Form and Movement 2009 Conference Proceedings: 188-195.

KNORR-CETINA, K. (1999) Epistemic Cultures, Cambridge, Harvard University Press.

LOZARES C., 2001. La actividad situada y/o el conocimiento socialmente distribuido Papers, 62, 2000, 97-131.

LOZARES C., VERD JM., MORENO S., BARRANCO O., MASSÓ M., 2004. El proceso de trabajo desde las perspectivas de la Actividad Situada y del Conocimiento Socialmente Distribuido. Cuadernos de Relaciones Laborales 22,1:67-87.

LOZARES C., TEVES L. Y MUNTANYOLA D., 2006. Prólogo: Del atomismo al relacionismo. La red socio-cognitiva como paradigma del cambio operado en la concepción de lo social y de la cognición [html] [pdf]. Monográfico Redes y Cognición . Ed por Lozares C., Teves L. y Lozares C. 2006. Las representaciones fácticas y cognitivas del relato de entrevistas biográficas: un análisis reticular del discurso [html] [pdf]. Monográfico Redes y Cognición . Ed por Lozares C., Teves L. y Muntanyola D. REDES, Revista hispana para el análisis de redes sociales. ISSN 157 9-0189. (Revista electrónica) www.redes-sociales.net Volumen 10.

LOZARES C. 2007 . "Aproximación a la cognición socialmente distribuida, CSD: un caso de estudio". En Lozares C. (coord.) Interacción, Redes Sociales y Ciencias Cognitivas. Granada: Ed. Camores. Cp IX pg. 277-313

LOZARES, C., VERD J.M., BARRANCO O., MORENO S., MASSO M. (2013) La complejidad y opacidad de las tareas auxiliares en los procesos de trabajo: un análisis etnográfico comparado en dos servicios hospitalarios. Cuadernos de Relaciones Laborales. Vol $31 \mathrm{~N}^{\circ} 1$ pg.221-242

MICHOTTE, A. (1954) La perception de la causalité. Louvain: Presses universitaires de Louvain.

MYERS, N. (2008) Crystallography Molecular Embodiments and the Body-work of Modeling in Protein, Social Studies of Science, 38, 2, 163-199.

MUNTANYOLA, D. (2009) Choreographing duets: gender differences in dance rehearsals, E-pisteme, 2, 2: 30-45.

MUNTANYOLA, D. 2010a. "Conocimiento experto y etnografía audiovisual: una propuesta teórico- metodológica." EMPIRIA. Revista de Metodología de Ciencias Sociales 20:109-133.

MUNTANYOLA, D. 2010b. "Danza y cognición: el proceso de creación coreográfica” J. Noya, M. Pérez \& F. del Val (Eds.) MUSYCA. Música, sociedad y creación artística. Madrid: Biblioteca Nueva.

MUNTANYOLA, D. 2011. "Los músicos y el coreógrafo: opiniones cruzadas en danza". Scherzo, 268: 24-29, Noviembre.

MUNTANYOLA, D. 2012. Expert knowledge and video-aided ethnography: a methodological account, Revue de synthèse, 133, 6, 1: 75-100. 
MUNTANYOLA, D. 2014a. Cognitive Account of Expertise: Why Rational Choice Theory is (often) a Fiction, Theory \& Psychology. DOI 10.1177/0959354313513510

MUNTANYOLA, D. 2014b. How Multimodality Shapes Creative Choice in Dance, Revista Internacional de Sociología, Vol.72, no 3, Septiembre-Diciembre, 563-582. DOI:10.3989/ris.2013.04.04

MUNTANYOLA, D. \& Kirsh, D. (2010) Marking as Physical Thinking: A Cognitive Ethnography of Dance. Proceedings of the IWCogSc-10 International Workshop on Cognitive Science, San Sebastian, 339-366.

NOICE, T. NOICE AND H. (1997) The Nature Of Expertise On Professional Acting: A Cognitive View, Mahwah, Lawrence Erlbaum Associates.

NOYA, J. DEL VAL, F, PEREZ, M. (Ed.): Musyca.: Music, society and artistic creativity. Madrid: Biblioteca Nueva.

POINCARE, H. (1908). Science and Method. London: Thomas Nelson \& Sons. Pylyshyn, Z. (2003) Seeing and Visualizing, Cambridge, MIT Press.

SAWYER, K. (2005) Social Emergence: Societies as Complex Systems, Cambridge University Press.

SIMMEL, G. (1908). Sociologie in Donald Levine (1971). Georg Simmel on Individuality and Social Forms. Chicago: University Chicago University Press.

SUCHMAN L. (1993). "Respuesta a la accion situada de Vera y Simon: una intepretacion simbólica". Cognitive Science, 17, 71-75.

WACQUANT, L. (2004) Body and Soul: Notes of an Apprentice Boxer, New York, Oxford University Press. 
